\title{
Mechanisms Involved in Lacunar Infarction and Their Role in Early Neurological Deterioration
}

\author{
Mi Ji Lee, MD, PhD* (D); Sujin Moon, BS ${ }^{\dagger}$ (D); Soohyun Cho, MD, MSc ${ }^{*}$ (D); Jong-Won Chung, MD, PhD ${ }^{*}$ (D); \\ Woo-Keun Seo, MD, PhD ${ }^{*}$ (D); Oh Young Bang, MD, PhD ${ }^{*}$ (D); Gyeong-Moon Kim, MD, PhD ${ }^{*}$ (D); \\ Chin-Sang Chung, MD, $\mathrm{PhD}^{\star}$
}

Department of Neurology, Samsung Medical Center, Sungkyunkwan University School of Medicine*, Seoul; School of Medicine, CHA University, Seongnam ${ }^{\dagger}$, Korea

Background: The mechanisms of lacunar infarction are still unclear and may be heterogenous. We aimed to investigate the presence of parent artery atheroma, cerebral vasodilatory function, and blood-brain barrier (BBB) breakdown and their role in the development of early neurological deterioration (END) in patients with acute lacunar infarction.

Methods: Patients with acute lacunar infarction prospectively underwent transcranial Doppler breath-holding test, contrast-enhanced fluid-attenuated inversion recovery magnetic resonance imaging, and magnetic resonance angiography with/without high-resolution vessel wall imaging to determine the cerebral vasomotor reactivity to carbon dioxide $\left(\mathrm{CO}_{2}\right.$-CVR) of the symptomatic artery, $\mathrm{BBB}$ breakdown, and the presence of branch atheromatous disease in the parent artery, respectively, before the development of END. We compared the presence of each mechanism between patients who developed END and those without END and the role in predicting END between mechanisms.

Results: Among the 41 patients included, three (7.3\%) experienced END. Reduced $\mathrm{CO}_{2}$-CVR, branch atheromatous disease, and widespread BBB breakdown were observed in eight (19.5\%), 10 (24.4\%), and 11 patients (26.8\%), respectively. Among these factors, $\mathrm{CO}_{2}$-CVR was significantly lower in patients with END than in those without END (breath-holding index, 0.64 [interquartile range, $0.47-0.70$ ] vs. 1.05 [0.81-1.33], $p=0.028$ ) and best predicted END (area under the curve $=0.889 ; 95 \%$ confidence interval, $0.779-0.999 ; p<0.001)$.

Conclusion: Cerebral vasodilatory dysfunction is associated with END in patients with acute lacunar infarction. Our study suggests that $\mathrm{CO}_{2}$-CVR testing has a role in the risk stratification of lacunar infarction, and a confirmatory study is required in the future.

J Neurosonol Neuroimag 2020;12(1):26-32

Key Words: Stroke; Lacunar infarction; Cerebral small vessel diseases; Transcranial Doppler ultrasonography; Blood-brain barrier
Received: Jan 13, 2020

Revised: Mar 16, 2020

Accepted: Mar 17, 2020

Correspondence:

Mi Ji Lee, MD, PhD

Department of Neurology, Samsung Medical Center, Sungkyunkwan University School of Medicine, 81 Irwon-ro, Gangnam-gu, Seoul 06351, Korea

Tel: +82-2-3410-3599

Fax: $+82-2-3410-1430$

E-mail: mijilee.md@gmail. com

\section{INTRODUCTION}

Lacunar infarction refers to a small subcortical infarct from the occlusion of a single perforating artery. Lacunar infarction accounts for up to $25 \%$ of all isch- emic strokes and causes significant morbidity. ${ }^{1,2}$ Early neurological deterioration (END) can occur in 20-30\% of patients with lacunar infarction during the first few days and lead to worse neurological outcomes. ${ }^{3}$

The mechanisms of lacunar infarction are still un- 
clear and significantly vary. Different from strokes attributed to large artery steno-occlusion or cardioembolism, there are no identifiable causes of lacunar infarction. Based on an autopsy series, lipohyalinosis has been recognized as a major pathophysiology of lacunar stroke and a result of hypertension. ${ }^{4}$ Microatheroma, atherosclerosis, arteriosclerosis, and arteriolosclerosis have been described in autopsy studies. ${ }^{2,4}$ Branch atheromatous disease (BAD) has been suggested as another mechanism of lacunar stroke. ${ }^{5}$

Recent studies focused on endothelial dysfunction as a proposed mechanism of lacunar stroke. Similar with systemic endothelium, cerebrovascular endothelium senses vasomotor (i.e., vasodilatory or vasoconstrictive) stimuli and modulates vasomotor response, particularly vasodilatory response occurring at the level of the distal arteries and arterioles. ${ }^{6}$ Additionally, cerebrovascular endothelium has a unique function as it forms the blood-brain barrier (BBB) together with pericytes, astrocyte foot processes, and tight-junction proteins at the level of the small arterioles and capillaries. ${ }^{7}$ Recent studies have shown the possible evidences of endothelial dysfunction in lacunar stroke, that is, reduced cerebrovascular reactivity to vasodilatory stimuli (e.g., carbon dioxide $\left[\mathrm{CO}_{2}\right]$ ) and widespread BBB leakage. .11 $^{8-11}$ Although it is unclear whether these phenomena are direct causes or mediators of lacunar stroke, endothelial dysfunction may play a role in the pathophysiology of lacunar stroke. ${ }^{12}$

The following questions are raised regarding lacunar infarction: What are the importance of the previously proposed mechanisms of lacunar infarction in real-world clinical settings and how do they overlap or relate with each other, and what are the clinical impacts of these mechanism? However, a study that comprehensively evaluates the previously suggested mechanisms of lacunar stroke and their clinical impacts has not been conducted yet. In this study, we comprehensively evaluated the markers of endothelial dysfunction ( $\mathrm{CO}_{2}$ cerebrovascular reactivity and $\mathrm{BBB}$ breakdown) and $\mathrm{BAD}$ in patients with acute lacunar infarction. We investigated the prevalence of each mechanism and tested their clinical impacts by comparing the role of each mechanism in predicting END.

\section{SUBJECTS AND METHODS}

\section{Patients}

We screened patients with acute ischemic stroke admitted to Samsung Medical Center between May 2017 and June 2018. Eligibility criteria were patients 1) aged $\geq 18$ years, 2) who visited within 7 days after symptom onset and had acute ischemic stroke confirmed by diffusion-weighted imaging, 3) who had a single subcortical infarction with a maximal diameter of $\leq 20 \mathrm{~mm}$ without $\geq 50 \%$ stenosis of the relevant artery and other evident mechanisms, and 4) whose symptomatic vessel was a perforator of the middle cerebral artery (MCA), posterior cerebral artery (PCA), or basilar artery (BA). Eligible patients were contacted by the investigators and excluded if they 1) had cognitive or cardiopulmonary impairment that prevented them from performing the breath-holding test, 2) had a contraindication for contrast-enhanced magnetic resonance imaging (MRI), and 3) refused to participate in this study. All included patients provided informed consents. After inclusion, patients dropped out if 1 ) they were unable to perform the breath-holding test or 2) other stroke mechanisms such as high-risk cardioembolic sources were documented during or after admission. The Institutional Review Board of Samsung Medical Center approved this study (IRB No. 2017-03-094). The data that support the findings of this study are available from the corresponding author upon reasonable request.

\section{Evaluation}

Vasodilatory function was measured by determining the cerebral vasomotor reactivity to $\mathrm{CO}_{2}\left(\mathrm{CO}_{2}\right.$-CVR) using transcranial Doppler (TCD) with the breath-holding maneuver per a protocol described elsewhere. ${ }^{13}$ In this study, the maximal mean flow velocities (MFVs) of the symptomatic vessel (MCA, PCA, or BA) were identified and monitored before and after a 30-s breath-holding maneuver. The breath-holding index (BHI) was calculated as the percentage increase in the MFV divided by the duration of breath holding (30 s) using the following equation: $\mathrm{BHI}=([\mathrm{MFV}-\mathrm{BH}-$ baseline $\mathrm{MFV}] /[$ baseline $\mathrm{MFV} \times 30 \mathrm{~s}]) \times 100$.

We also determined the presence of BBB breakdown 
using delayed contrast-enhanced fluid-attenuated inversion recovery MRI. Our protocol and its rationale have been published elsewhere. ${ }^{14}$ Gadolinium leakage into the cerebrospinal fluid space was evaluated by investigators and classified into local and widespread BBB breakdown.

BAD was suspected in patients with mild $(<50 \%)$ stenosis in the corresponding segment of the parent artery. In patients having normal-appearing parent arteries, high-resolution vessel wall imaging was performed to investigate the presence of atheroma causing BAD. Finally, BAD was defined based on the presence of parent artery stenosis in magnetic resonance angiography or an atheroma in high-resolution MRI. Perfusion deficits were evaluated by delayed time to peak in perfusion-weighted imaging.

\section{Outcome}

END was defined as $\geq 2$ increase in the National Institutes of Health Stroke Scale within 7 days after admission. ${ }^{15}$ In this study, we only regarded neurological worsening from progressive stroke as END. ${ }^{3}$ Follow-up neuroimaging was performed in all patients experiencing neurological worsening. Neurological worsening from new stroke in other vascular territory, hemorrhagic transformation, seizure, edema, or other medical conditions was carefully differentiated from progressive stroke.

\section{Statistical analyses}

Data are presented as a number (\%) or median (interquartile range [IQR]). Demographics, characteristics of stroke, and major study results were compared between patients with vs. without END. The predictive power of each variable was tested by the area under the curve (AUC) of the receiver operating characteristic (ROC) curve. The optimal cutoff point was determined by calculating the Youden's index. Statistical analyses were performed using Stata 15.0 (StataCorp. 2017. Stata Statistical Software: Release 15, StataCorp LLC, College Station, TX, USA). Differences with a two-tailed $p$-value $<0.05$ were considered statistically significant.

\section{RESULTS}

The study flowchart is shown in Fig. 1. From 710 patients screened during the study period, 82 patients were eligible for this study. Among the eligible patients, we recruited 46 patients. After five patients were dropped out, 41 patients were analyzed. Out of the 41 patients included in the study, the median interval from the symptom onset to admission was 1 day (IQR, O-2), and MRI and TCD studies were performed at the median of o (IQR, O-1) and 2 days (IQR, 1-3) after admission, respectively.

Demographics and characteristics of the 41 included patients are summarized in Table 1. END was observed in three patients $(7.3 \%)$ at the median of 2.5 days (1.4-6.0) after symptom onset. Demographics and baseline characteristics were not different between patients with vs. without END.

Results from study investigations are demonstrated in Table 2. All the investigations were completed before

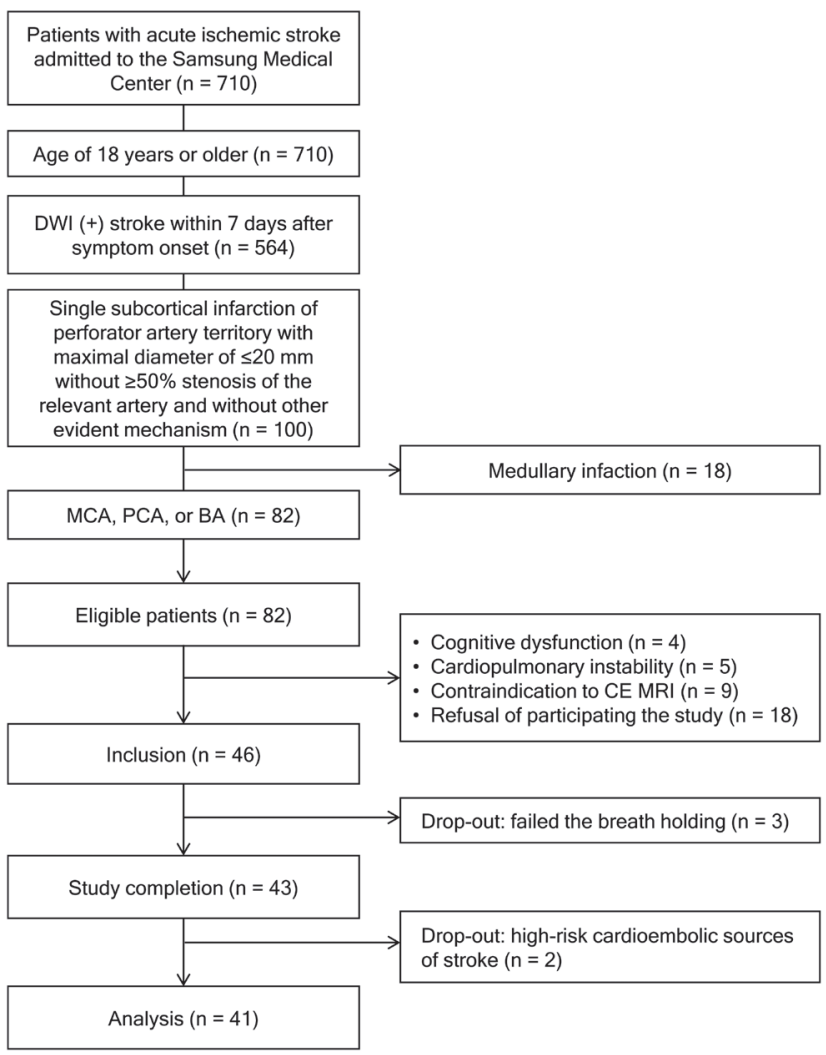

FIG. 1. Study flowchart. DWI; diffusion-weighted image, MCA; middle cerebral artery, PCA; posterior cerebral artery, BA; basilar artery, $\mathrm{CE}$; contrast-enhanced, $\mathrm{MRI}$; magnetic resonance imaging. 
the development of END. Among the mechanisms investigated, BBB breakdown was most prevalent (30.0\%), and $\mathrm{BAD}$ was observed in $24.4 \%$ of patients. BHI was significantly lower in patients who developed END than those without END (0.64 [0.47-0.70] vs. 1.05 [0.81-1.3], $\mathrm{p}=0.028)$. BAD and widespread BBB breakdown were more common in patients who developed END compared to patients who did not develop END, although the difference was statistically insignificant (66.7\% vs. $21.1 \%, p=0.142 ; 33.3 \%$ vs. $13.5 \%$, $p=0.394)$. Diffusion-perfusion mismatch was insignificantly different between the two groups.

TABLE 1. Demographics and characteristics of the study participants

\begin{tabular}{|c|c|c|c|c|}
\hline & Total $(n=41)$ & No $\operatorname{END}(n=38)$ & $\operatorname{END}(n=3)$ & $p$-value \\
\hline Age & $69(63-74)$ & $69(60-74)$ & $73(65-90)$ & 0.316 \\
\hline NIHSS & $2(1-3)$ & $2(1-3)$ & $3(1-6)$ & 0.359 \\
\hline Male sex & $22(53.7)$ & $20(52.6)$ & $2(66.7)$ & $>0.999$ \\
\hline Symptomatic vessel & & & & $>0.999$ \\
\hline Middle cerebral artery & $23(56.1)$ & $21(55 \cdot 3)$ & $2(66.7)$ & \\
\hline Posterior cerebral artery & $8(19 \cdot 5)$ & $8(21.1)$ & $o(0)$ & \\
\hline Basilar artery & $10(24.4)$ & $9(23.7)$ & $1(33.3)$ & \\
\hline Parent artery & & & & $>0.999$ \\
\hline No stenosis & $37(90.2)$ & $34(89.5)$ & $3(100)$ & \\
\hline Mild stenosis $(<50)$ & $4(9.8)$ & $4(10.5)$ & $\mathrm{o}(\mathrm{o})$ & \\
\hline Hypertension & $36(87.8)$ & $33(86.8)$ & $3(100)$ & $>0.999$ \\
\hline Diabetes & $16(39.0)$ & $14(36.8)$ & $2(66.7)$ & 0.550 \\
\hline Dyslipidemia & $23(56.1)$ & $21(55 \cdot 3)$ & $2(66.7)$ & $>0.999$ \\
\hline Smoking & & & & 0.671 \\
\hline Never & $27(65.9)$ & $24(63.2)$ & $3(100)$ & \\
\hline Current & $10(24.4)$ & $10(26.3)$ & o (o) & \\
\hline Ex-smoker $(<5$ years $)$ & $4(9.8)$ & $4(10.5)$ & $\mathrm{o}(0)$ & \\
\hline Previous stroke/TIA & $6(14.6)$ & $5(13.2)$ & $1(33 \cdot 3)$ & 0.386 \\
\hline Coronary artery disease & $4(9.8)$ & $4(10.5)$ & $o(0)$ & $>0.999$ \\
\hline
\end{tabular}

Values are presented as median (interquartile range) or number (\%).

END; early neurological deterioration, NIHSS; National Institutes of Health Stroke Scale, TIA; transient ischemic attack, NA; not assessed.

TABLE 2. Comparison of mechanisms involved in lacunar stroke between patients with vs. without early neurological deterioration

\begin{tabular}{|c|c|c|c|c|}
\hline & Total $(n=41)$ & No $\operatorname{END}(n=38)$ & $\operatorname{END}(n=3)$ & $p$-value \\
\hline Breath-holding index of the symptomatic vessel & $0.99(0.75-1.31)$ & $1.05(0.81-1.33)$ & $0.64(0.47-0.70)$ & 0.028 \\
\hline Branch atheromatous disease & $10(24.4)$ & $8(21.1)$ & $2(66.7)$ & 0.142 \\
\hline \multicolumn{5}{|l|}{ PWI } \\
\hline DWI-PWI mismatch & $11(26.8)$ & $10(26.3)$ & $1(33 \cdot 3)$ & $>0.999$ \\
\hline \multicolumn{5}{|l|}{ BBB breakdown } \\
\hline Any & $12(30.0)$ & $11(29.7)$ & $1(33.3)$ & $>0.999$ \\
\hline Widespread & $6(15 \cdot 0)$ & $5(13.5)$ & $1(33 \cdot 3)$ & 0.394 \\
\hline
\end{tabular}

Values are presented as median (interquartile range) or number (\%).

END; early neurological deterioration, PWI; perfusion-weighted image, DWI; diffusion-weighted image, BBB; blood-brain barrier. 
Comparative ROC analysis showed that the CVR of the symptomatic artery best predicted the development of END (AUC $=0.889$ [95\% confidence interval, 0.7790.999], p<0.001; Fig. 2). The optimal cutoff point was 0.74 , with a sensitivity of $84.9 \%$ and a specificity of $100.0 \%$.

Based on the findings above, we defined a significant vasodilatory dysfunction as $\mathrm{BHI}$ less than 0.74. Together with the widespread BBB breakdown and $\mathrm{BAD}$, any abnormalities were observed in 16 patients (39.0\%). The number of patients having overlapping abnormalities is demonstrated in Fig. 3.

\section{DISCUSSION}

In this study, we evaluated several mechanisms associated with lacunar infarction. While other major subtypes of ischemic stroke are classified based on causative mechanisms, only lacunar infarction has been defined not by causative findings but by clinico-radiological manifestations. ${ }^{16}$ Our study showed that the following possible mechanisms contributed to the development of lacunar infarction: significant vasodilatory dysfunction in one-fifth, BAD in one-fourth, and BBB breakdown in one-third of patients. Our study result highlights that the pathophysiologies of lacunar infarction significantly vary and are demonstrated by several appropriate neuroimaging and neurophysiology tests.

Our study showed that vasodilatory dysfunction as measured by reduced cerebrovascular reactivity to $\mathrm{CO}_{2}$

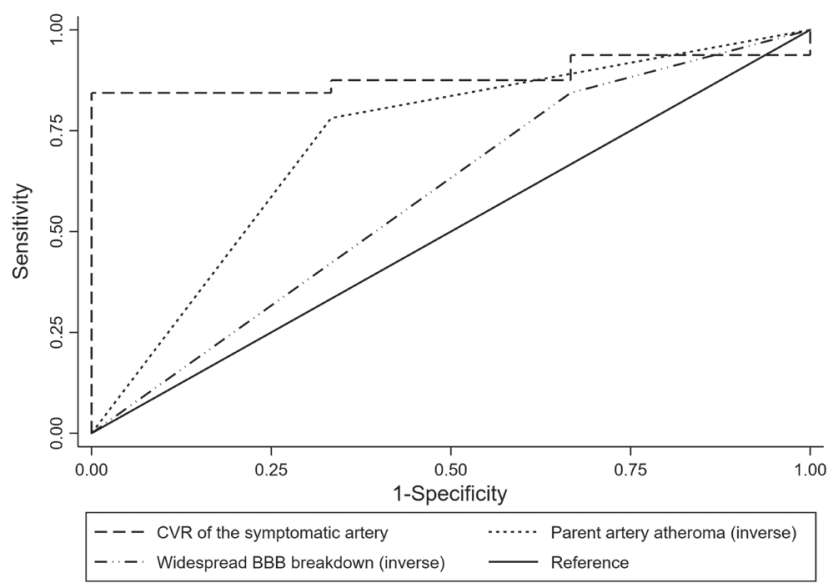

FIG. 2. Comparative receiver operating characteristic curve analysis. CVR; cerebrovascular reactivity, BBB; blood-brain barrier. is associated with END in patients with lacunar infarction. Cerebral blood flow is regulated by metabolic (e.g., mental activation or therapeutic hypothermia), chemical (e.g., $\mathrm{CO}_{2}$, oxygen, pH, or lactic acid), myogenic (e.g., intraluminal pressure), and, to a minimal degree, neurogenic factors (e.g., sympathetic or parasympathetic activities). ${ }^{17}$ Besides the myogenic factor, the endothelium is the key mediator of vasomotor regulation among the metabolic and chemical factors. ${ }^{6,17}$ For the chemical regulation, the endothelium senses bloodborne signals and responds by releasing a number of autocrine and paracrine substances. ${ }^{6}$ Nitric oxide is the major neuropeptide that causes vasodilation and acts on vascular smooth muscle cells, which are effectors of vasomotion. ${ }^{18,19}$ Reduced cerebrovascular reactivity to $\mathrm{CO}_{2}$ indicates impaired nitric oxide-mediated endothelial function, which is the common pathway for most of the metabolic and chemical regulations of cerebral blood flow.

In this study, vasodilatory dysfunction in non-stenotic symptomatic arteries preceded the development of END in lacunar infarction and had a significant power in predicting END. To date, parent artery stenosis, $\mathrm{BAD}$, and perfusion deficits have been reported as predictors of END in patients with lacunar infarction..$^{20,21}$ Among these predictors of END, our findings suggest that CVR has a prognostic role in the risk stratification of lacunar infarction. CVR is an indicator of cerebrovascular reserve of the territory of the tested artery. ${ }^{22}$ We hypothesized that reduced CVR might cause a reduc-

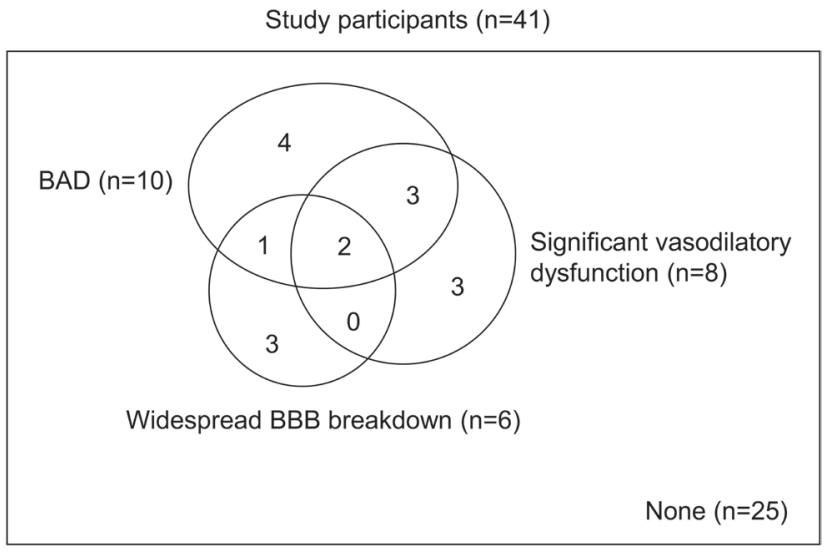

FIG. 3. Prevalence of suggested mechanisms of lacunar infarction in the study participants. BAD; branch atheromatous disease, BBB; blood-brain barrier. 
tion of vasodilation and reduced collateral flows to the ischemic area, which can further lead to an increase in the infarct core. Although our study was preliminary, it can provide background information for a confirmatory study before implementing the TCD-CVR test as a routine test in the prognostication of lacunar infarction. Additionally, our study suggests that treatment strategies to improve vasodilatory function in preventing and treating END in patients with lacunar infarction are required.

Our study has the following strengths: it uses a prospective study design, it comprehensively evaluates lacunar infarction, and it uses advanced imaging techniques when assessing lacunar infarction. However, this study also has the following limitations: it is a preliminary study, it has insufficient evidences to confirm a hypothesis, a possibility of overfitting due to a small number of patients is observed in this study, and a low incidence of END is observed in this study.

In conclusion, our study suggests mechanisms involved in lacunar infarction and the possible role of CVR in the risk stratification of patients with acute lacunar infarction. Further study using a larger cohort is required to confirm our findings.

\section{Acknowledgements}

This work was supported by the grant of the Korean Society of Neurosonology and the National Research Foundation of Korea grant funded by the Korean government (MSIP) (Nos. 2017R1A2B4007254 and 2017R1A2B2009086).

\section{Conflicts of interest}

No potential conflicts of interest relevant to this article was reported.

\section{REFERENCES}

1. Norrving B. Long-term prognosis after lacunar infarction. Lancet Neurol. 2003;2:238-245.

2. Regenhardt RW, Das AS, Lo EH, Caplan LR. Advances in understanding the pathophysiology of lacunar stroke: a review. JAMA Neurol. 2018;75:1273-1281.

3. Del Bene A, Palumbo V, Lamassa M, Saia V, Piccardi B, Inzitari D. Progressive lacunar stroke: review of mechanisms, prognostic features, and putative treatments. Int J Stroke. 2012;7:321-329.

4. Fisher CM. The arterial lesions underlying lacunes. Acta Neuropathol. 1968;12:1-15.

5. Caplan LR. Intracranial branch atheromatous disease: a neglected, understudied, and underused concept. Neurology. 1989;39:1246-1250.

6. Deanfield JE, Halcox JP, Rabelink TJ. Endothelial function and dysfunction: testing and clinical relevance. Circulation. 2007;115:1285-1295.

7. Abbott NJ, Rönnbäck L, Hansson E. Astrocyte-endothelial interactions at the blood-brain barrier. Nat Rev Neurosci. 2006;7:41-53.

8. Wardlaw JM, Doubal F, Armitage P, Chappell F, Carpenter T, Muñoz Maniega S, et al. Lacunar stroke is associated with diffuse blood-brain barrier dysfunction. Ann Neurol. 2009;65:194-202.

9. Deplanque D, Lavallee PC, Labreuche J, Gongora-Rivera F, Jaramillo A, Brenner D, et al. Cerebral and extracerebral vasoreactivity in symptomatic lacunar stroke patients: a case-control study. Int J Stroke. 2013;8:413-421.

10. Molina C, Sabín JA, Montaner J, Rovira A, Abilleira S, Codina A. Impaired cerebrovascular reactivity as a risk marker for first-ever lacunar infarction: a case-control study. Stroke. 1999;30:2296-2301.

11. Knottnerus IL, Ten Cate H, Lodder J, Kessels F, van Oostenbrugge RJ. Endothelial dysfunction in lacunar stroke: a systematic review. Cerebrovasc Dis. 2009;27:519-526.

12. Stevenson SF, Doubal FN, Shuler K, Wardlaw JM. A systematic review of dynamic cerebral and peripheral endothelial function in lacunar stroke versus controls. Stroke. 2010;41:e434-e442.

13. Lee MJ, Park BY, Cho S, Park H, Chung CS. Cerebrovascular reactivity as a determinant of deep white matter hyperintensities in migraine. Neurology. 2019;92:e342-e350.

14. Lee MJ, Cha J, Choi HA, Woo SY, Kim S, Wang SJ, et al. Blood-brain barrier breakdown in reversible cerebral vasoconstriction syndrome: implications for pathophysiology and diagnosis. Ann Neurol. 2017;81:454-466.

15. Siegler JE, Boehme AK, Kumar AD, Gillette MA, Albright KC, Martin-Schild S. What change in the National Institutes of Health Stroke Scale should define neurologic deterioration in acute ischemic stroke? J Stroke Cerebrovasc Dis. 2013;22:675-682.

16. Ay H, Furie KL, Singhal A, Smith WS, Sorensen AG, Koroshetz WJ. An evidence-based causative classification system 
for acute ischemic stroke. Ann Neurol. 2005;58:688-697.

17. Benarroch EE, Daube JR, Flemming KD, Westmoreland BF. Mayo clinic medical neuroscience: organized by neurologic systems and levels. 5th ed. Rochester: Mayo Foundation for Medical Education and Research, 2008;462-469.

18. Furchgott RF, Zawadzki JV. The obligatory role of endothelial cells in the relaxation of arterial smooth muscle by acetylcholine. Nature. 1980;288:373-376.

19. Förstermann U, Münzel T. Endothelial nitric oxide synthase in vascular disease: from marvel to menace. Circulation. 2006;113:1708-1714.
20. Jeong HG, Kim BJ, Yang MH, Han MK, Bae HJ. Neuroimaging markers for early neurologic deterioration in single small subcortical infarction. Stroke. 2015;46:687-691.

21. Poppe AY, Coutts SB, Kosior J, Hill MD, O’Reilly CM, Demchuk AM. Normal magnetic resonance perfusion-weighted imaging in lacunar infarcts predicts a low risk of early deterioration. Cerebrovasc Dis. 2009;28:151-156.

22. Liu P, De Vis JB, Lu H. Cerebrovascular reactivity (CVR) MRI with $\mathrm{CO}_{2}$ challenge: a technical review. Neuroimage. 2019;187:104-115. 